\title{
Editorial
}

\section{Frauen und Gesundheit (oder die Gesundheit der Frauen?)}

Frauen haben eine höhere Lebenserwartung, fühlen sich subjektiv aber weniger gesund als Männer, «beanspruchen» das Gesundheitswesen mehr (oder benützen es besser?), bezahlen - zumindest in der Schweiz - höhere Krankenkassenprämien: Widersprüche oder Beschreibung eines komplexen Systems, in welchem die einzelnen, messbaren Gegebenheiten sich gegenseitig beeinflussen? Das vorliegende Heft möchte eine Standortbestimmung der Erforschung der Geschlechtsunterschiede in Gesundheit und Krankheit versuchen. Die Betrachtung dieser Unterschiede gibt Hinweise darauf, wie sie im Sinne der verbesserten Gesundheit für alle verkleinert werden könnten, was jeder vom andern Geschlecht übernehmen könnte. Diese Überlegung mag den Leser bei der Lektüre der vorliegenden Nummer leiten: Die Mortalitätsunterschiede (beschrieben für Deutschland) und Veränderungen in der Krebsmortalität in der Schweiz zeigen, dass diese Unterschiede nur teilweise genetisch bedingt sind, ihre zeitlichen Veränderungen weisen deutlich auf Umwelt- und Verhaltenseinflüsse hin. Mehrere Arbeiten befassen sich mit den Verhaltenseinflüssen: Neben den bekannten Risikofaktoren, die für Männer und Frauen gleichartig - wenn auch unterschiedlich stark - wirken, haben Frauen durch die Reproduktionsfunktion ein weiteres Risiko. Neben der Frage, wie weit die hormonale Kontrazeption die cardiovaskuläre Mortalität beeinflusst(e), interessiert auch die Früherfassung der bisher häufigsten Karzinome der Frau.

Die Analyse der Verminderung von Risikofaktoren, Vergleiche zwischen eigener Wahrnehmung und untersuchter Verteilung weisen darauf hin, dass Frauen Gesundheitsbelange eher beachten und auch eher gewillt sind, entsprechende Konsequenzen zu ziehen. Erklärt dies die immer wieder beschriebene höhere Konsultationshäufigkeit von Frauen, welche in der Schweiz zu erhöhten Krankenkassenprämien für Frauen führt? Ein Artikel lässt noch andere Vermutungen aufkommen: Frauen werden bei der Erstkonsultation bereits in einem kleinen Kollektiv länger untersucht und öfter wieder einbestellt. Weitere Forschung sollte diesen Geschlechtsunterschieden nachgehen.

Die vorliegende Nummer will Geschlechtsunterschiede in Gesundheit und Krankheit (soweit sie untersucht sind) darstellen. Mag sie als Anregung dienen, das Grundanliegen der Sozial- und Präventivmedizin auch aus diesem Blickwinkel zu stärken: Die Veränderung geschlechtsspezifischer Rollenverständnisse sollte auch zur Übernahme der gesundheitsfördernden Verhaltensweise des jeweils anderen Geschlechtes führen. 


\section{Editorial}

\section{Les femmes et la santé (ou la santé des femmes?)}

Les femmes ont une espérance de vie supérieure à celle des hommes, mais se jugent en moins bonne santé: elles se sentent plus concernées par la santé (ou peut-être savent-elles mieux l'apprécier) et leurs primes de caisse-maladie sont plus élevées que celles des hommes: contradictions ou fondement d'un système complexe dans lequel tous les éléments interagissent?

La présente livraison de la Revue fait le point sur les différences liées au sexe dans les domaines de la santé et de la maladie. La nature de ces différences donne d'utiles indications sur ce que chaque sexe peut apprendre de l'autre pour améliorer son comportement en matière de santé et pour diminuer les différences observées.

L'évolution des différences de mortalité générale en Allemagne et de la mortalité cancéreuse en Suisse suggèrent que les facteurs génétiques n'expliquent qu'en partie les disparités entre les sexes: c'est au contraire le style de vie et l'environnement qui déterminent largement ces différences. Plusieurs travaux sont consacrés à l'influence du style de vie; ils font remarquer qu'outre les facteurs agissant dans les deux sexes (avec un impact éventuellement différent), il existe des risques spécifiquement liés à la fonction reproductive des femmes. Ces risques concernent d'une part l'influence de la contraception hormonale sur la mortalité cardiovasculaire et, d'autre on s'intéresse au diagnostique précoce du cancer du sein qui est le plus fréquent des cancers féminins.

L'étude du contrôle des facteurs de risque, les comparaisons entre leur prise en charge et leur répartition effective montrent que les femmes sont plus sensibles que les hommes à la notion de santé et sont mieux disposées à en tirer toutes les conséquences. Ce comportement explique-t-il le taux de consultations ambulatoires notoirement plus élevé chez les femmes (ce qui, en Suisse, implique une prime de caissemaladie plus élevée)? L'un des travaux présentés suggère d'autres explications encore: les patientes seraient examinées plus longuement que les hommes lors de la première consultation, et seraient plus souvent reconvoquées. Ces différentiels mériteraient d'être ultérieurement investigués.

Ce numéro présente les différences liées au sexe dans les domaines de la santé et de la maladie, pour autant que ces différences soient investiguées; il souhaite renforcer la prise en compte d'un aspect particulier en médecine sociale et préventive: la transformation des rôles respectifs des hommes et des femmes dans notre société devrait également faire adopter les comportements positifs à l'égard de la santé inspirés de l'autre sexe.

Ursula Ackermann-Liebrich, Bâle 